Dr Nicholas Levy

Consultant in Anaesthesia and Peri-operative Medicine

Department of Anaesthesia and Peri-operative Medicine

West Suffolk NHS Foundation Trust

Hardwick Lane

Bury St Edmunds

Suffolk, UK

Tel: $+44(0) 1284712819$

Fax: +44 (0)1284 712819

Email: Nicholas.levy@wsh.nhs.uk

Invited editorial

Restoration of function: the holy grail of peri-operative care

Nicholas Levy ${ }^{1}$, Michael PW Grocott ${ }^{2}$, Dileep N Lobo ${ }^{3}$

1. Consultant, Department of Anaesthesia and Peri-operative Medicine, West Suffolk NHS Foundation Trust, Bury St Edmunds, Suffolk

2. Professor of Anaesthesia and Critical Care Medicine, Southampton NIHR Biomedical Research Centre, University Hospitals Southampton NHS Foundation Trust / University of Southampton, Southampton, UK

3. Professor of Gastrointestinal Surgery, Nottingham Digestive Diseases Centre, National Institute for Health Research (NIHR) Nottingham Biomedical Research Centre, Nottingham University Hospitals and University of Nottingham, Queen's Medical Centre, Nottingham NG7 2UH, UK and MRC/ARUK Centre for Musculoskeletal Ageing Research, School of Life Sciences, University of Nottingham, Queen's Medical Centre, Nottingham, NG7 2UK, UK

Conflict of Interest: None of the authors has a direct conflict of interest to declare. DNL has received unrestricted research funding from BBraun and speaker's honoraria from Fresenius Kabi, BBraun, Shire and Baxter Healthcare for unrelated work. MPWG has received unrestricted research funding from Sphere Medical Ltd (UK) and Pharmacosmos Ltd (UK) and serves on the medical advisory board of Sphere Medical Ltd.

Key Words: enhanced recovery after surgery; functional recovery; pre-optimisation; quality of recovery; peri-operative care; restoration of function; quality measures; patient care

Author contributions: All authors contributed equally to this article

Correspondence to : Dr. Nicholas Levy

Word count : 1849

Twitter Handles : @ @nicholasalevy @DL08OMD @mike_grocott 


\section{Restoration of function: the holy grail of peri-operative care}

In this collaborative supplement between Anaesthesia and the British Journal of Surgery, there are reviews of enhanced recovery after surgery in both the elective [1] and the emergency patient [2] by Kehlet, a surgeon from Denmark. These are complimented by reviews on the quality of recovery by Myles [3], and on patient-centred outcomes by Ladaha and Wijeysundera [4], anaesthetists from Australia and Canada respectively.

As the originator of the concept of enhanced recovery after surgery (ERAS)/fast track surgery, Kehlet describes the challenges of introducing ERAS programmes [1]. These challenges persist despite the well-documented success of ERAS programmes in improving surgical outcomes as defined by shortening length of stay, readmission rates, medical complications, and healthcare costs. He also articulates the pathophysiological challenges of surgery, and the recurring theme is promoting return of normal function. In fact, Kehlet first identified the importance of promoting restoration of function in 1994, when he was devising the concept of multi-modal analgesia, and he discussed the triple aim of post-operative pain relief in providing subjective comfort; attenuation of the stress response and to enhance restoration of function by allowing the patient to breathe, cough and move more easily [5]. This remarkable insight from 25 years ago is only now beginning to be fully appreciated. Crucially, despite an absence of evidence of efficacy [6] or safety [7], many centres continue to administer post-operative opioid analgesia in response to unidimensional pain intensity scores $[4,8]$, rather than function. This is despite a paucity of evidence to support the discredited "Pain as the Fifth Vital Sign" campaign [8]. In fact, in 1994, Kehlet proposed the concept that pain should be managed to promote function [5]. Only recently has the necessity to titrate additional opioid analgesia to promote restoration of function been more widely recognised [8-12]. Titration of additional opioid to function allows the balance between the beneficial effects of analgesia in relieving pain and promoting restoration of function, and the well-recognised adverse effects of 
excessive opioids including sedation; opioid-induced ventilatory impairment (OIVI); dependence and delayed return of gastrointestinal function after surgery to be achieved more safely [8-13]. This concept has now been endorsed by several organisations including the Joint Commission, the health services regulatory body of the United States [9], and the Australian and New Zealand College of Anaesthetists [10]. The Functional Activity Score is a simple but effective measurement tool that is beginning to gain traction to promote this goal [8, 10-12].

In the past 25 years patient demographics have evolved, as have patient expectations. Thus, the proportion of elderly patients with multiple comorbidities and polypharmacy presenting for both elective and emergency surgery has increased and will continue to do so [4]. This presents particular challenges and Kehlet discusses the evolving field of optimisation of function (pre-optimisation) before surgery [1]. Whilst prehabilitation encompasses the concept of pre-optimisation of the patient's physical and psychological state [14], comorbidity management describes the optimisation of care for pre-existing medical conditions (e.g. diabetes and anaemia). It is now being increasingly appreciated that one of the major current challenges facing both anaesthetists and surgeons is the identification of the group of patients that will not achieve restoration of function/return of independence and do not have either the physiological or psychological reserve to benefit from preoptimisation or surgery $[4,15]$. Thus, all patients now require a thorough pre-operative assessment [4], which is often and increasingly being supplemented by cardiopulmonary exercise stress testing [13]. As well as the physical conditions including frailty that may preclude or delay return of function [4], there is growing awareness that pre-existing psychological states, including, for example, chronic pain states, patients on pre-existing opioids and pain catastrophisers may also preclude a restoration of function [1]. Moreover, there is a realisation that many patients may prioritise restoration of function including preservation of independence over longevity, and for them, an acceptable outcome entails improvements in health, rather than simply avoidance of death or an overt 
complication [4]. Shared decision making allows these dilemmas to be articulated [16] and involves a detailed and individualised discussion with the patient concerning the likelihood of potential harms, including non-return to base line function and independence, whilst discussing the benefits of surgery and any alternatives to the proposed surgical procedure. Critical to enabling these processes is the reconfiguration of pre-operative pathways to allow sufficient time for assessment and discussion; pathway redesign is becoming an important theme in peri-operative care [17].

In the narrative on optimising recovery after emergency laparotomy, Foss and Kehlet discuss the challenges facing the peri-operative team to improve the outcome of the patient requiring an emergency laparotomy, and lament the lack of robust scientific data to guide practice [2]. However, they emphasise the importance of promoting restoration of function through early nutrition and mobilisation to minimise post-operative complications.

Meanwhile, Myles [3] reviews the tools that are used to assess the quality of recovery after surgery. He describes how these have moved on from traditional indices such as length of stay, and rate of major and minor complications to a greater emphasis on patient-reported outcome measures (PROMs). He also emphasises that global measures of a patient's quality of recovery, avoidance of post-operative morbidities, early hospital discharge to home (without re-admission), and longerterm disability-free survival can define post-operative recovery better. But what do we mean by recovery? Previously it could have been considered to have occurred by the time of discharge from the recovery unit. This is an outmoded perspective and explains why a more useful and precise term for the "recovery ward" is now post anaesthetic care unit (PACU). Others may define recovery as being equivalent to readiness for hospital discharge, which explains why length of stay has been considered an important indicator for surgical outcomes. In reality, full recovery only occurs once normal baseline function has been restored/superseded and adverse symptoms have resolved. Thus, 
there are at least three stages to recovery (discharge from recovery, discharge from hospital and return to baseline), and each stage needs to be satisfactorily achieved for complete recovery to occur [18]. The quality of recovery (QoR) scores tools developed by Myles are powerful research tools and facilitate robust comparisons between different techniques and different institutions, and are mainly designed to evaluate the effectiveness of the second and third stages of recovery. They provide a valid and useful measure of recovery that facilitates evaluation of surgical and anaesthetic techniques that either need to be promoted or discarded.

As well as discussing the tools that are available to record the quality of recovery, and noting that some are now being used to facilitate shared decision making, Myles [3] emphasises that one of the most important functions of analysing recovery data is to promote continuous quality improvement. Internationally, the Multicenter Perioperative Outcomes Group (MPOG) is conducting such work primarily in North America. Whilst in the UK, the National Institute of Academic Anaesthesia's (NIAA) Health Services Research Centre, working on behalf of the Royal College of Anaesthetists and the Royal College of Surgeons of England, in collaboration with a number of other societies and professional bodies in 2016 established the Perioperative Quality Improvement Programme (PQIP). PQIP is the UK's peri-operative continuous quality improvement project and its aim is to examine the peri-operative care of patients undergoing major non-cardiac surgery and measure complication rates, failure to rescue and patient-reported outcomes, with the aim of improving patient outcomes by reducing variation in processes of care and supporting implementation of best practice. Data are collected at 5 distinct time points (pre-op, day 1 and 3 post-operative, and then at 6 and 12 months post-operatively). To date over 124 hospitals are involved and over 21,800 patients have been recruited. Clearly acknowledging that restoration of function is a key component of recovery, one of the five prioritised PQIP national quality improvement opportunities is the measuring and promoting the return of Drinking, Eating and Mobilising (DrEaMing). The attraction of comparing DrEaMing 
rates is that the components of DrEaMing are binary. In addition, if a patient is "DrEaMing", it implies adequate pain control; an absence of post-operative ileus; an absence of orthostatic hypotension; and adequate fluid balance, haemoglobin, cardiac output and respiratory function [19]. Furthermore, as immobilisation is a recognised risk factor for thromboembolic events, promoting mobilisation has clear preventative advantages. Moreover, once patients are eating and drinking, they are no longer generally dependant on either intravenous fluids or intravenous drugs, which is vital to prevent iatrogenic complications from intravenous fluid administration. In addition, patients with a variety of pre-existing medical conditions including diabetes and Parkinson's disease can revert to their usual medication faster, with less potential for harm.

Despite the attractiveness of using DrEaMing as a surrogate measure of quality of peri-operative care and restoration of function, it still needs to be validated in this role, and it is hoped that the PQIP team will be able to demonstrate its predictive utility in relation to validated positive outcomes. Moreover, if early DrEaMing is shown to be beneficial, intra-operative techniques can be adjusted to promote restoration of major organ function further [20]. This may include increased reliance on regional anaesthesia techniques as a part of procedure specific postoperative pain management (Prospect) [21, 22]. However, whilst DrEaMing is a binary measure summarising return of intestinal, cardio-respiratory and muscular function, it does not record return of cognitive function. It is increasingly being recognised that peri-operative neurocognitive disorders (PNCD) are a major cause of morbidity [23], and unlike the QoR scores, DrEaMing does not incorporate it. Thus, despite the simplicity of DrEaMing, it cannot replace currently validated scoring systems.

In addition, none of the scoring systems reviewed by either Myles [3] or Ladha and Wijeysundera [4] are designed to examine explicitly the incidence or causes of the major iatrogenic peri-operative complication of the $21^{\text {st }}$ century namely prescribed opioid dependence [24]. The incidence of 
persistent post-operative opioid use ranges from $0.6 \%$ to $26 \%$ for opioid-naïve patients and from $35 \%$ to $77 \%$ for patients with previous opioid exposure [25]. Therefore, there is now an urgent need for quality of recovery scoring systems to be updated or augmented to examine the incidence and causes of persistent post-operative opioid use. The benefits of surgery must not be allowed to be negated by the subsequent harm and reduction in function caused by persistent opioid use.

In conclusion, over the past quarter of a century there has been a gradual recognition that promotion of restoration of function is vital to a full recovery and is an important outcome that matters both to patients and health services. The challenges for the next 25 years will include improvements in collaborative working between surgeons, anaesthetists and other health professionals to promote restoration of function further. This must be coupled with the continuous evolution of scoring systems so that surgical patients at risk of not achieving restoration of normal homeostatic, physical and psychological function, including independence, can be identified earlier. This evolution of practice will enable the individualisation of care to facilitate restoration of function and full recovery through shared decision making, prehabilitation, peri-operative co-morbidity management, and rehabilitation to achieve the best outcomes for our patients. This is the goal of peri-operative care. 


\section{References}

1. Kehlet $\mathrm{H}$. Enhanced postoperative recovery: good from afar - but far from good? Anaesthesia 2020; this supplement.

2. Foss NB, Kehlet $\mathrm{H}$. Challenges in optimizing recovery after emergency laparotomy. Anaesthesia 2020; this supplement.

3. Myles PS. More than just morbidity and mortality - quality of recovery and long-term functional recovery after surgery. Anaesthesia 2020; this supplement.

4. Ladha KS, Wijeysundera DN. Patient-centred outcomes after hospital discharge - what matters? Anaesthesia 2020; this supplement.

5. Kehlet H. Postoperative pain relief--what is the issue? British Journal of Anaesthesia 1994; 72: $375-8$.

6. Mularski RA, White-Chu F, Overbay D, Miller L, Asch SM, Ganzini L. Measuring pain as the 5 th vital sign does not improve quality of pain management. Journal of General Internal Medicine 2006; 21: 607-12.

7. Vila H, Jr., Smith RA, Augustyniak MJ, et al. The efficacy and safety of pain management before and after implementation of hospital-wide pain management standards: is patient safety compromised by treatment based solely on numerical pain ratings? Anesthesia and Analgesia 2005; 101: 474-80.

8. Levy N, Sturgess J, Mills P. "Pain as the fifth vital sign" and dependence on the "numerical pain scale" is being abandoned in the US: Why? British Journal of Anaesthesia 2018; 120 : 435-8.

9. The Joint Commission. $\mathrm{R}^{3}$ Report. Pain assessment and management standards for hospitals. 2017. Available at: https://www.jointcommission.org/assets/1/18/R3_Report_Issue_11_Pain_Assessment_2_1 1_19_REV.pdf (Accessed 10 August 2019).

10. Australian and New Zealand College of Anaesthetists. Statement on principles for identifying and preventing opioid-induced ventilatory impairment (OIVI). 2018. Available at: http://www.anzca.edu.au/resources/endorsed-guidelines/oivi-statement (Accessed 12 August 2019).

11. Levy N, Mills $P$, Rockett M. Post-surgical pain management: time for a paradigm shift. British Journal of Anaesthesia 2019; 123: e182-e6.

12. Tong YG, Konstantatos AH, Cheng Y, Chai L. Improving pain management through addition of the functional activity score. Australian Journal of Advanced Nursing 2018; 35: 52-60.

13. McEvoy MD, Scott MJ, Gordon DB, et al. American Society for Enhanced Recovery (ASER) and Peri-operative Quality Initiative (POQI) joint consensus statement on optimal analgesia within an enhanced recovery pathway for colorectal surgery: part 1-from the preoperative period to PACU. Perioperative Medicine (London) 2017; 6: 8.

14. Scheede-Bergdahl C, Minnella EM, Carli F. Multi-modal prehabilitation: addressing the why, when, what, how, who and where next? Anaesthesia 2019; 74 Suppl 1: 20-6. 
15. Bilimoria KY, Liu Y, Paruch JL, et al. Development and evaluation of the universal ACS NSQIP surgical risk calculator: a decision aid and informed consent tool for patients and surgeons. Journal of the American College of Surgeons 2013; 217: 833-42 e1-3.

16. Sturgess J, Clapp JT, Fleisher LA. Shared decision-making in peri-operative medicine: a narrative review. Anaesthesia 2019; 74 Suppl 1: 13-9.

17. Grocott MPW, Plumb JOM, Edwards M, Fecher-Jones I, Levett DZH. Re-designing the pathway to surgery: better care and added value. Perioperative Medicine (London) 2017; 6: 9.

18. Bowyer AJ, Royse CF. Postoperative recovery and outcomes--what are we measuring and for whom? Anaesthesia 2016; 71 Suppl 1: 72-7.

19. Levy N, Mills P, Mythen M. Is the pursuit of DREAMing (drinking, eating and mobilising) the ultimate goal of anaesthesia? Anaesthesia 2016; 71: 1008-12

20. Stubbs D, Levy N, Moonesinghe SR. Good intra-operative anaesthesia is more than an 'airway, breathing, circulation, drugs with a three, two and a one'. Anaesthesia 2019 [Epub ahead of print] doi:10.1111/anae.14809.

21. Joshi GP, Van de Velde M, Kehlet H; PROSPECT Working Group Collaborators. Development of evidence-based recommendations for procedure-specific pain management: PROSPECT methodology. Anaesthesia 2019; 74: 1298-1304.

22. Albrecht $\mathrm{E}$, Chin KJ. Advances in regional anaesthesia and acute pain management. Anaesthesia 2020; this supplement.

23. White $S$, Griffiths R, Baxter M, et al. Guidelines for the peri-operative care of people with dementia: Guidelines from the Association of Anaesthetists. Anaesthesia 2019; 74: 357-72.

24. Kharasch ED, Brunt LM. Peri-operative opioids and public health. Anesthesiology 2016; 124 : 960-5.

25. Kent ML, Hurley RW, Oderda GM, et al. American Society for Enhanced Recovery and Perioperative Quality Initiative-4 Joint Consensus Statement on Persistent Postoperative Opioid Use: Definition, Incidence, Risk Factors, and Health Care System Initiatives. Anesthesia and Analgesia 2019; 129: 543-52. 